\title{
Effect of finite system size on the thermodynamics of hot and magnetized hadron resonance gas
}

\author{
Debasis Atta* and Snigdha Ghosh \\ Government General Degree College Kharagpur-II, \\ Paschim Medinipur - 721149, West Bengal, India,
}

E-mail: debasisa906@gmail.com, snigdha.physics@gmail.com

The thermodynamic properties of a non-interacting ideal Hadron Resonance Gas (HRG) of finite volume have been studied in the presence of an external magnetic field. The inclusion of background magnetic field in the calculation of thermodynamic potential is done by the modification of the dispersion relations of charged hadrons in terms of Landau quantization. The generalized Matsubara prescription has been employed to take into account the finite size effects in which a periodic (anti-periodic) boundary conditions is considered for the mesons (baryons). We find significant effects of the magnetic field as well as system size on the temperature dependence of energy density, longitudinal and transverse pressure especially in low temperature regions.

\footnotetext{
*** Particles and Nuclei International Conference - PANIC2021 ***

*** 5 - 10 September, $2021 * * *$

*** Online ***
}

${ }^{*}$ Speaker 


\section{INTRODUCTION}

The study of hot and dense strongly interacting matter created in relativistic heavy ion collision (HIC) at RHIC and LHC has been of great research interest. A non-central HIC can produce magnetic field of the order of $\sim 10^{18}$ Gauss or more, (which is comparable to the QCD energy scale), so that various exotic phenomena like chiral magnetic effect, magnetic catalysis, inverse magnetic catalysis could take place [1]. Bulk thermodynamic properties or the phase structure of QCD can be analyzed in a HIC experiments. The numerical lattice QCD (LQCD) based calculations [2] is an approach to study the QCD thermodynamics, but is limited to the low baryon density region of the QCD phase diagram due to its 'sign' problem. Beside that, the hadron resonance gas (HRG) model [3] is a statistical thermal model for studying the QCD thermodynamics at finite temperature, baryon density as well as external magnetic field $[4,5]$. Interestingly, at low temperature and small baryon density, the results from HRG model agrees well with the LQCD.

In the calculation of thermodynamic quantities, one generally assumes the system size to be infinite. However, in the HIC experiments, the created fireball has finite volume $\left(\sim \mathrm{few} \mathrm{fm}^{3}\right)$. So, it is justified to consider the boundary effects in the calculation of thermodynamical quantities pertaining to the HIC [5]. In this work, the thermodynamic quantities like energy density, longitudinal and transverse pressure and magnetization of an ideal HRG of finite size in presence of external magnetic field have been calculated. The formalism of generalized Matsubara prescription [6] will be used to incorporate the finite size effect whereas the effect of external magnetic field will enter through the Landau quantization of the dispersion relations of charged hadrons.

\section{FORMALISM}

Let us start with the standard expression of the thermodynamic potential (density) $\Omega$ of an ideal HRG at zero-magnetic field in infinite volume $(B=0$ and $L=\infty)$ as [5]

$$
\Omega=-T \sum_{i \in\{\text { hadrons }\}} g_{i} a_{i} \int \frac{d^{3} k}{(2 \pi)^{3}} \ln \left(1+a_{i} e^{-\beta \omega_{k}^{i}}\right)
$$

where $g_{i}=\left(2 s_{i}+1\right)$ is the spin degeneracy of hadron $i$ having spin $s_{i}, a_{i}=-(-1)^{2 s_{i}}=+1$ if $i \in$ \{baryons $\}$ and -1 if $i \in\{$ mesons $\}, \beta=1 / T$ is the inverse temperature and $\omega_{k}^{i}=\sqrt{\vec{k}^{2}+m_{i}^{2}}$ is the single particle energy of hadron $i$ having mass $m_{i}$.

From Eq. (1), all the other thermodynamic quantities like isotropic pressure $P=-\Omega$, energy density $\varepsilon=-T^{2} \frac{\partial}{\partial T}\left(\frac{\Omega}{T}\right)$, the entropy density $s=(\varepsilon+P) / T$ etc. can be calculated.

Next, we consider HRG at $B \neq 0$ and $L=\infty$, with an external magnetic field $\vec{B}=B \hat{z}$ in the positive- $\hat{z}$ direction. The single particle energies of charged hadrons will now be Landau quantized as $\omega_{k l s}^{i}=\sqrt{k_{z}^{2}+\left\{2 l+1-2 s \operatorname{sign}\left(e_{i}\right)\right\}\left|e_{i}\right| B+m_{i}^{2}}$ where $s=-s_{i},-s_{i}+1, \cdots, s_{i}$ and $l=0,1,2, \cdots$, where, $e_{i}$ is the electronic charge of hadron $i$. It is to be noted that, here $l$ is related to the orbital angular momentum quantum number. The thermodynamic potential of ideal HRG of Eq. (1) in presence of external magnetic field modifies to

$$
\Omega_{B}=\Omega_{\text {neutral }}-T \sum_{i \in\left\{\begin{array}{l}
\text { charged } \\
\text { hadrons }
\end{array}\right\}} a_{i} \sum_{l=0}^{\infty} \sum_{s=-s_{i}}^{s_{i}} \frac{\left|e_{i}\right| B}{2 \pi} \int_{-\infty}^{\infty} \frac{d k_{z}}{2 \pi} \ln \left(1+a_{i} e^{-\beta \omega_{k l s}^{i}}\right)
$$


where, the first term on the right hand side corresponds to the contribution from the neutral hadrons which are not affected by the external magnetic field in the leading order. In the presence of external magnetic field, the pressure becomes anisotropic and have different values along the longitudinal and transverse direction with respect to the direction of the magnetic field [7]. The longitudinal pressure $\left(P_{\|}\right)$and transverse pressure $\left(P_{\perp}\right)$ can now be calculated respectively from $P_{\|}=-\Omega_{B}$ and $P_{\perp}=P_{\|}-\left(\frac{\partial P_{\|}}{\partial B}\right) B$.
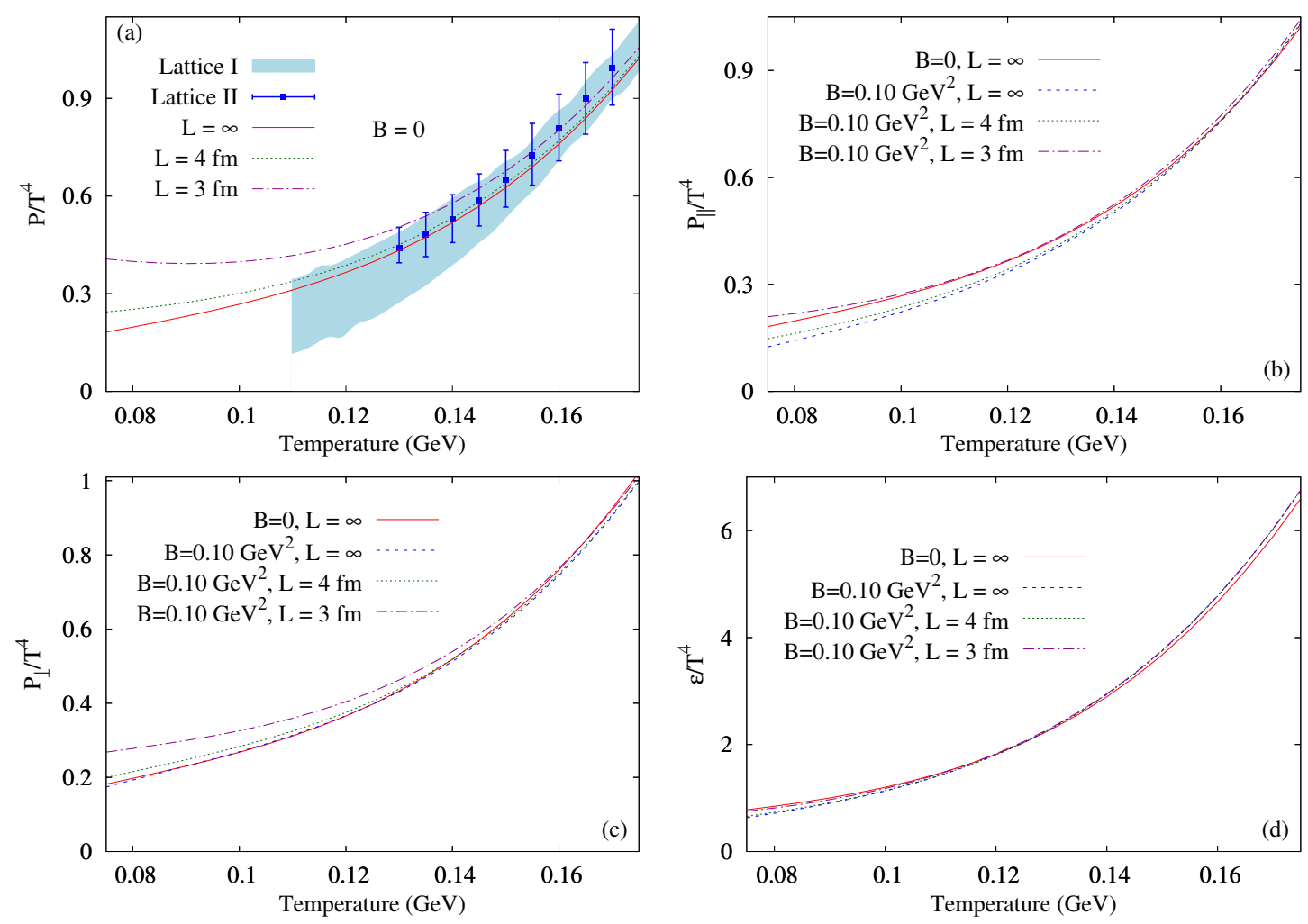

Figure 1: The variation of (a) $P / T^{4}$, (b) $P_{\|} / T^{4}$, (c) $P_{\perp} / T^{4}$, and, (d) $\varepsilon / T^{4}$ as a function of temperature ( $T$ ) for different values of system size $(L)$ and external magnetic field $B$. For comparison, the results of Lattice QCD calculations from Ref. [8] and Ref. [9] are also shown as Lattice I and Lattice II respectively in sub-figure-(a)

Until now, we have considered the system size to be infinite. To take into account the finite-volume effect in HRG thermodynamics, we employ the formalism of generalized Matsubara prescription as discussed in Ref. [6]. For this, we consider our system to be a cube of length $L$ so that, the spatial coordinates lie in the interval $x^{i} \in[0, L]$. As a consequence of the generalized Matsubara prescription, the momentum integral at $B=0$ will have to be replaced with sum over discrete Matsubara modes as $\int \frac{d^{3} k}{(2 \pi)^{3}} f(\vec{k}) \rightarrow \frac{1}{L^{3}} \sum_{n_{x}, n_{y}, n_{z}=-\infty} f\left(\vec{k}_{n_{x} n_{y} n_{z}}\right)$ where, $\vec{k}_{n_{x}, n_{y}, n_{z}}=\frac{2 \pi}{L}\left[\left(n_{x}+b\right) \hat{x}+\left(n_{y}+b\right) \hat{y}+\left(n_{z}+b\right) \hat{z}\right]$. Here the parameter $b$ can be chosen appropriately to consider periodic or anti-periodic boundary condition in the compactified spatial coordinates. We consider periodic boundary condition for the mesons and anti-periodic boundary condition for the baryons [6]. Hence, in our calculation, we choose the parameter $b=0$ for Mesons and $b=1 / 2$ for Baryons. In the presence of external magnetic field, the following Matsubara pre- 
scription has been used for a magnetized HRG of finite size: $\int_{-\infty}^{\infty} \frac{d k_{z}}{2 \pi} f\left(k_{z}\right) \rightarrow \frac{1}{L} \sum_{n_{z}=-\infty}^{\infty} f\left(k_{z ; n_{z}}\right)$, where, $k_{z ; n_{z}}=\frac{2 \pi}{L}\left(n_{z}+b\right)$.

\section{NUMERICAL RESULTS AND DISCUSSIONS}

The dimensionless scaled values of $\frac{P}{T^{4}}, \frac{\varepsilon}{T^{4}}, \frac{P_{\|}}{T^{4}}$, and, $\frac{P_{\perp}}{T^{4}}$ as a function of temperature for both zero and non-zero magnetic field for different system size have been depicted in Fig. 1 Here both the pressure and energy density increase monotonically with increase in temperature. This is due to the enhancement of thermal phase space of hadron owing to the increase of their average thermal energy. The results of Fig. 1 from HRG model for $L=\infty$ are in excellent agreement with the corresponding LQCD results. We find, the magnetic field makes the pressure of the system anisotropic, and the pressure has different values in the longitudinal and transverse direction. Moreover, significant effects of the magnetic field as well as system size on the temperature dependence of energy density, longitudinal and transverse pressure are observed. The effects of system size and magnetic field are found to be more in low temperature regions.

\section{References}

[1] D. Kharzeev, K. Landsteiner, A. Schmitt and H.-U. Yee, eds., Strongly Interacting Matter in Magnetic Fields, vol. 871. 2013, 10.1007/978-3-642-37305-3.

[2] P. de Forcrand and O. Philipsen, The Chiral critical line of $N(f)=2+1$ QCD at zero and non-zero baryon density, JHEP 01 (2007) 077 [hep-lat/0607017].

[3] J. Cleymans and K. Redlich, Chemical and thermal freezeout parameters from 1-A/GeV to 200-A/GeV, Phys. Rev. C 60 (1999) 054908 [nucl-th/9903063].

[4] G. Endrödi, QCD equation of state at nonzero magnetic fields in the Hadron Resonance Gas model, JHEP 04 (2013) 023 [1301.1307].

[5] A. Bhattacharyya, R. Ray, S. Samanta and S. Sur, Thermodynamics and fluctuations of conserved charges in a hadron resonance gas model in a finite volume, Phys. Rev. C 91 (2015) 041901 [1502.00889].

[6] L. M. Abreu, E. B. S. Corrêa, C. A. Linhares and A. P. C. Malbouisson, Finite-volume and magnetic effects on the phase structure of the three-flavor Nambu-Jona-Lasinio model, Phys. Rev. D 99 (2019) 076001 [1903.09249].

[7] M. Strickland, V. Dexheimer and D. P. Menezes, Bulk Properties of a Fermi Gas in a Magnetic Field, Phys. Rev. D 86 (2012) 125032 [1209. 3276].

[8] S. Borsanyi, Z. Fodor, C. Hoelbling, S. D. Katz, S. Krieg and K. K. Szabo, Full result for the QCD equation of state with 2+1 flavors, Phys. Lett. B 730 (2014) 99 [1309.5258].

[9] НотQCD collaboration, Equation of state in ( 2+1)-flavor QCD, Phys. Rev. D 90 (2014) 094503 [1407.6387]. 